\title{
Nuclear Bragg Scattering of Synchrotron Radiation Pulses in a Single-Reflection Geometry
}

\author{
R. Rüffer, E. Gerdau, and R. Hollatz \\ II. Institut für Experimentalphysik, Universität Hamburg, D-2000 Hamburg 50, Federal Republic of Germany
}

and

J. P. Hannon

Physics Department, Rice University, Houston, Texas 77001

(Received 2 March 1987)

\begin{abstract}
We have observed the delayed time spectrum of coherently scattered synchrotron-radiation pulses following a single nuclear Bragg reflection in ${ }^{57} \mathrm{Fe}$-yttrium iron garnet. A new quantum-beat spectrum with more hyperfine information was obtained by our taking $B_{\text {int }}$ perpendicular to the scattering plane $\left(k_{0}, k_{f}\right)$. The speedup of coherent decay at the Bragg angle due to "enhancement," and the quantumbeat modulation of the coherent decay rate, are shown.
\end{abstract}

PACS numbers: $76.80 .+\mathrm{y}, 07.85 .+\mathrm{n}, 42.10 . \mathrm{Qj}, 75.50 . \mathrm{Gg}$

The possibility of monochromatizing synchrotron radiation to $\sim 10^{-8} \mathrm{eV}$ in the $1-\AA \mathrm{x}$-ray region with resonant Mössbauer filters, and the observation of quantum beats in the coherently scattered radiation from nuclei excited by synchrotron-radiation pulses, have been reported in two previous papers. ${ }^{1,2}$ Both of these experiments used a double nuclear Bragg-diffraction geometry. We have now observed the delayed time spectrum using a single-reflection geometry. Also, by our taking the orientation for the applied magnetic field perpendicular to the scattering plane, a new quantum-beat spectrum ${ }^{3}$ with more hyperfine information was produced. In addition, the results give an interesting illustration both of the quantum-beat modulation of the coherent decay rate, and of the speedup of the coherent decay at the Bragg angle due to "enhancement." The single-reflection geometry simplifies the analysis of the time spectrum, and, more importantly, makes it possible to carry out experiments with other crystals which lack the macroscopic perfection required for double-reflection experiments.

The experimental arrangement was the same as in the previous experiments ${ }^{1,2}$ except that now the nuclear Bragg monochromator was a single ${ }^{57} \mathrm{Fe}$-yttrium iron garnet crystal, and we introduced a silver-surfaced total-reflection mirror following the Si premonochromator. By this means it was possible nearly to suppress the $\lambda / 2$ component and higher harmonics transmitted by the premonochromator. Consequently, the light produced in the plastic scintillator was drastically reduced, and there were relatively few pulses which exceeded the pulse height belonging to the $14.4-\mathrm{keV}$ radiation. The total rate of accidental events was thus about $0.1 \mathrm{~Hz}$. However, the resonant counting rate $(1 \mathrm{~Hz})$ was no larger than in the previous double-reflection experiments. This was caused by the silver mirror, which reduced the cross section and increased the angular divergence of the beam. Nevertheless, even with a counting rate of only $1 \mathrm{~Hz}$, it is possible within a day to determine a hyperfine interaction with a precision which can hardly be achieved in a normal absorption experiment with a strong source. The result of our measurements is shown in Fig. 1. The solid line indicates the theoretical calculation, which we discuss below.

The observations were made with use of a single (002) nuclear Bragg reflection in ${ }^{57} \mathrm{Fe}$-yttrium iron garnet. The [100] crystal axis lay in the scattering plane $\left(\mathbf{k}_{0}, \mathbf{k}_{f}\right)$ of the incident and reflected beams, but the direction of the internal hyperfine field $B_{\text {int }}$ was rotated from the [100] to the [010] direction, perpendicular to the scattering plane. The scattering arises as before from the nuclei located at the $d_{1}$ and $d_{2}$ sites within the unit cell, ${ }^{2,4}$ but the linear $\hat{\sigma}$ and $\hat{\pi}$ polarizations are very nearly eigenpolarizations for the scattering, with $\hat{\sigma}$ coupling to the eight " $\Delta J_{z} "= \pm 1$ transitions, and $\hat{\pi}$ coupling to the four $\Delta J_{z}=0$ transitions. Figure 2 illustrates the relative positions of the resonance energies. The incident syn-

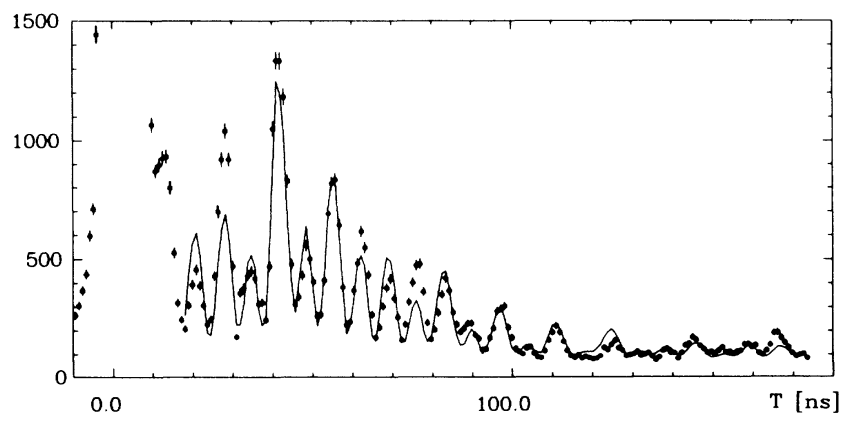

FIG. 1. Time spectrum of the coherently scattered radiation. The solid line is the calculated theoretical spectrum, plus a constant background contribution. The ratio of delayed to prompt counts is about $1 \times 10^{-3}$. The data were accumulated over one day. 
chrotron radiation is elliptically polarized with a dominant contribution of more than $90 \% \hat{\sigma}$ polarization.

The nature of the quantum-beat spectrum is easily understood by use of the Born-approximation limit. For incident $\hat{\sigma}$ polarization, the $\pm \frac{1}{2} \leftrightarrow \pm \frac{3}{2}$ transitions have relative strength 1 and the $\mp \frac{1}{2} \leftrightarrow \pm \frac{1}{2}$ transitions have strength $\frac{1}{3}$, with the contributions from the $d_{1}$ and $d_{2}$ sites being $180^{\circ}$ out of phase. With these amplitudes and phases, and with the particular symmetry of the splittings shown in Fig. 2, the eight component waves (with 28 possible difference frequencies) sum up to give a simple amplitude-modulated time spectrum. For a single reflection, the $\hat{\sigma}$ contribution is

$$
I_{\sigma}(t) \propto e^{-\Gamma t / \hbar} \sin ^{2}\left(\frac{1}{2} \Omega_{E} t\right)\left|\cos \left[\frac{1}{2} \Omega_{M}(1,6) t\right]-\frac{1}{3} e^{-i \Delta \Omega t} \cos \left[\frac{1}{2} \Omega_{M}(3,4) t\right]\right|^{2} .
$$

In this formula we have dropped for simplicity the higher-order modifications which take into account the $\epsilon$ shifts ${ }^{5}$ of the transition energies given in Fig. 2, which do not change the characteristic features of the curve. The electric field gradient splitting is $\hbar \Omega_{E}=3 \Delta / 4$ between adjacent $d_{1}$ and $d_{2}$ lines; $\hbar \Omega_{M}(1,6)$ is the magnetic splitting between the two outer \pm 1 transitions, $\hbar \Omega_{M}(3,4)$ is the magnetic splitting between the two inner \pm 1 transitions, and $\hbar \Delta \Omega=\Delta / 4$ is the shift of the

$$
\left|\cos \left[\frac{1}{2} \Omega_{M}(1,6) t\right]-\frac{1}{3} \exp (-i \Delta \Omega t) \cos \left[\frac{1}{2} \Omega_{M}(3,4) t\right]\right|^{2}
$$

superimposes a fast ringing on the slowly developing envelope. The pattern is dominated by the leading term, $\cos ^{2}\left[\frac{1}{2} \Omega_{M}(1,6) t\right]$, which arises from the interference between the strong outer lines, and gives a dominant fast beat period $2 \pi / \Omega_{M}(1,6)=6.8 \mathrm{~ns}$ at room temperature. ${ }^{6}$ The contribution arising from the interference between the weaker $(3,4)$ lines, $\frac{1}{9} \cos ^{2}\left[\frac{1}{2} \Omega_{M}(3,4) t\right]$, has only a

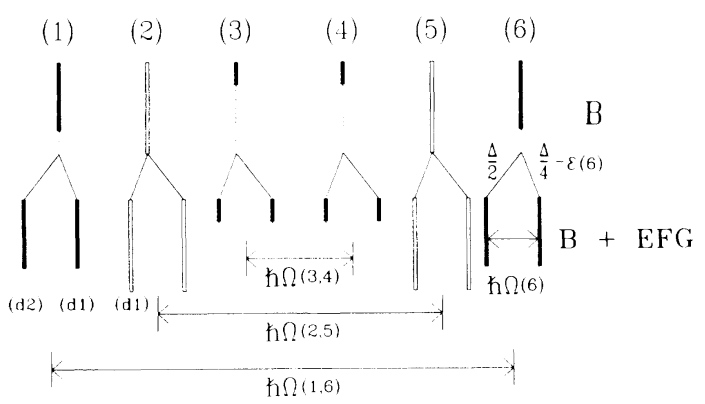

FIG. 2. Hyperfine splittings between the eight $\Delta J_{z}= \pm 1$ transitions (filled symbols) and between the four $\Delta J_{z}=0$ transitions (open symbols) at the $d_{1}$ and $d_{2}$ sites when $\mathbf{B}_{\text {int }}$ is along the $[010]$ crystal axis. Here the transitions are (1) $\triangleq\left[+\frac{1}{2}\right.$ $\left.\leftrightarrow+\frac{3}{2}\right],(2) \wedge\left[+\frac{1}{2} \leftrightarrow+\frac{1}{2}\right]$, (3) $\triangleq\left[+\frac{1}{2} \leftrightarrow-\frac{1}{2}\right]$, (4) $\triangleq\left[-\frac{1}{2}\right.$ $\left.\leftrightarrow+\frac{1}{2}\right],(5) \hat{=}\left[-\frac{1}{2} \leftrightarrow-\frac{1}{2}\right]$, (6) $\hat{=}\left[-\frac{1}{2} \leftrightarrow-\frac{3}{2}\right]$. In the drawing the $\epsilon$ shifts of the full Hamiltonian are added. This causes corrections of the order $10^{-3}$ to the magnetic interactions and of a few percent to the electric interactions which are unimportant for the present discussion of the time response. From Ref. 4 and Ref. 2 the room-temperature values are $\hbar \Omega(1,6) \approx \hbar \Omega_{M}(1,6)=126.9 \Gamma, \quad \hbar \Omega(3,4) \approx \hbar \Omega_{M}(3,4)$ $=20.0 \Gamma, \hbar \Omega(2,5) \approx \hbar \Omega_{M}(2,5)=73.5 \Gamma, \hbar \Omega(i)+\epsilon(i)=\hbar \Omega_{E}$ $=6.54 \Gamma, \Delta=9.17 \Gamma, \epsilon(1)=\epsilon(3)=\epsilon(5)=0.30 \Gamma$ and $\epsilon(2)=\epsilon(4)$

$=\epsilon(6)=-0.27 \Gamma$ with $\Gamma=4.665 \times 10^{-9} \mathrm{eV}$. center of the four $(3,4)$ lines from the center of the four $(1,6)$ lines.

The leading factor, $\exp (-\Gamma t / \hbar) \sin ^{2}\left(\frac{1}{2} \Omega_{E} t\right)$, is the same factor which occurs in the previously used orientation of $\mathbf{B}_{\text {int. }}$ As discussed in Ref. 2, this gives a slowly developing overall modulation, which arises from the interference between the adjacent electric field gradient split lines.

The factor

small effect on the pattern. A very perceptible modulation is, however, caused by the interference between the $(1,6)$ lines and the $(3,4)$ lines. This contribution,

$$
-\frac{2}{3} \cos (\Delta \Omega t) \cos \left[\frac{1}{2} \Omega_{M}(3,4) t\right] \cos \left[\frac{1}{2} \Omega_{M}(1,6) t\right],
$$

alternately adds and subtracts from the leading contribution $\cos ^{2}\left[\frac{1}{2} \Omega_{M}(1,6) t\right]$ every fast beat period, giving a "high-low" pattern, with the order depending on the sign of the factor $\cos (\Delta \Omega t) \cos \left[\frac{1}{2} \Omega_{M}(3,4) t\right]$. This factor changes sign at odd multiples of $\pi / \Omega_{M}(3,4)=20$ ns and at odd multiples of $\pi / 2 \Delta \Omega=76 \mathrm{~ns}$. In addition to the new high-low modulation, another interesting feature is that the fast beats are much more pronounced than in the previous spectrum. ${ }^{2}$ This occurs because now the fast beats are produced by the interference between the equally strong (1) and (6) lines, so that essentially complete destructive interference occurs, whereas previously the interference was between the unequally strong (1) and (4) lines [and between the (3) and (6) lines].

We note that the present quantum-beat spectrum contains more hyperfine information because the frequencies $\Omega_{M}(1,6), \Omega_{M}(3,4)$, and $\Omega_{E}$ determine all three hyperfine parameters $g_{0}, g_{1}$, and $\Delta$. In the previous experiment, the fit only depended on $g_{0}+g_{1}$ and $\Delta$.

For incident $\hat{\pi}$ polarization, the $\pm \frac{1}{2} \rightarrow \pm \frac{1}{2}$ transitions all have relative strength $\frac{4}{3}\left(\frac{2}{3}\right.$ for the square of the Clebsch-Gordan coefficient, and a factor of 2 for the improved polarization coupling, relative to the $\hat{\sigma}$ coupling), with the $d_{1}$ and $d_{2}$ contributions again being $180^{\circ}$ out of phase. The $\hat{\pi}$ contribution is then simply ${ }^{5}$

$$
I_{\pi}(t) \propto \frac{4}{3} e^{-\Gamma t / \hbar} \sin ^{2}\left(\frac{1}{2} \Omega_{E} t\right) \cos ^{2}\left[\frac{1}{2} \Omega_{M}(2,5) t\right],
$$

where, as shown in Fig. $2, \Omega_{M}(2,5)$ is the magnetic splitting between the $\Delta J_{z}=0$ transitions. This has the 
same slow modulation as before, and now a single fast beat frequency with a period of $11.5 \mathrm{~ns}$. In our experimental arrangement, $I_{\pi}$ gives only a contribution of about $6 \%$ to the total $I(t)$. However, if the crystal were to be rotated to a "crossed geometry" with reference to the plane of the synchrotron radiation, then $\hat{\pi}$ would be the dominant incident polarization.

For theoretical simulation of the data, we have used the dynamical theory calculation outlined in Ref. 2 using elliptical polarization with a mixing angle of about $15^{\circ}$ for a single-reflection from yttrium iron garnet (YIG) with $B_{\text {int }}$ perpendicular to $\left(\mathbf{k}_{0}, \mathbf{k}_{f}\right){ }^{7}$ Integration over the angular divergence of the incident beam, which we have taken to be a Gaussian spread with $\sigma=12 \mu \mathrm{rad}$ centered at peak Bragg reflectivity, leads to the solid line in Fig. 1. Although the simulation is generally good, there are observable discrepancies between calculation and experiment in the amplitudes of several of the peaks, such as those at 30, 70, and $77 \mathrm{~ns}$. Relative amplitudes of the peaks are sensitive to the rocking angle, angular divergence, and distribution of polarization of the incident beam, and effects such as small asymmetries in the internal fields or weak inhomogeneous broadening, as might be caused by strains or uneven heating. Precise values for the YIG hyperfine parameters will be published after further investigation of these points. Finally, we note that our measurements illustrate two points of theoretical interest. Following excitation by the prompt synchrotron pulse, the nuclear system is in a phased collective excited state. ${ }^{3}$ For excitation at the Bragg angle, the subsequent single-photon decay rate exhibits both a speedup, due to "coherent enhancement," and a quantum-beat modulation.

The quantum-beat modulation of the coherent decay rate arises from the interference between component waves of different frequencies emitted from the various nuclear transitions, and produces a periodic modulation of the probability for photon emission into the coherent channel. A striking example of this behavior in the present experiment is the initial complete suppression of the coherent decay, followed by a slow buildup of constructive interference, leading to a delayed peak response at a characteristic time $\pi / \Omega_{E}$. This effect is due to interference between adjacent transitions from the $d_{1}$ and $d_{2}$ sites, which are emitting waves of frequencies $\omega_{0}$ and $\omega_{0}+\Omega_{E}$. For the coherent decay at the (002) Bragg condition, the waves are initially $180^{\circ}$ out of phase because of the spatial separation between the $d_{1}$ and $d_{2}$ sites, leading to the initial suppression of coherent decay.

The second feature, the speedup of coherent decay, is a consequence of the "enhancement effect." As pointed out by Trammell, ${ }^{8,9}$ when a resonant crystal is excited at the Bragg angle, there is an enhancement of the coherent elastic radiative width, and correspondingly, the time response for coherent scattering is speeded up. This is a simple consequence of the constructive interference occurring at the Bragg angle between component waves of the same frequency emanating from the various nuclear sites. A direct observation of the speedup using time-coincidence techniques has been made by Smirnov, Shvyd'ko, and Realo. ${ }^{10}$ The exact nature of coherent enhancement varies, depending on whether the scattering system is a thin crystal film or a thick crystal, whether one well-isolated resonance or several close-lying strongly interfering resonances occur (which is the situation in YIG), and whether excitation is at the Bragg angle or at grazing incidence. For the scattering of synchrotron pulses, the resulting speedup at the Bragg angle for the case of a thick crystal and an isolated resonance has been treated by Kagan, Afanas'ev, and Kohn, ${ }^{11}$ and the grazing-incidence case has been discussed by Hannon $e t$ al. ${ }^{12}$ For synchrotron scattering from YIG, the coherent speedup directly manifests itself in a shift of the peak response of the slowly developing quantum-beat envelope. The location of the peak response is determined by the product of the exponential decay by the initial sinusoidal increase [Eq. (1)], and gives a peak response at

$$
t_{\max }=\left(2 / \Omega_{E}\right) \arctan \left(\hbar \Omega_{E} / \Gamma\right)=62 \mathrm{~ns} .
$$

The effect of coherent enhancement, which leads to a more rapid decay than $\exp (-\Gamma t / \hbar)$, is to pull in the peak response to 43 ns. Although the actual response is more complex, ${ }^{7}$ the effect here is approximately a simple replacement of $\Gamma \rightarrow \Gamma+\Gamma_{c} \approx 4 \Gamma$ ( $c$ denotes coherent) in the Born-approximation expression. There, $4 \Gamma$ is the approximate half-width of the frequency response of the reflectivity of YIG about each of the $d_{1}-d_{2}$ resonance pairs. Since the increasing width is due to coherent radiative decay, this corresponds to an enhancement of the radiative decay width by a factor of $\left(\Gamma+\Gamma_{c}-\Gamma_{\alpha}\right) / \Gamma_{\gamma}$ $\approx 29$. In Fig. 3 we draw the quantum-beat envelope for $\Gamma$ and $4 \Gamma$ together with the measured time spectrum. In

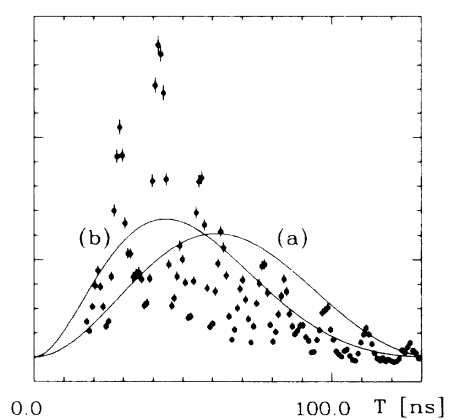

FIG. 3. Speedup of the time response in YIG. The envelope function, $\exp (-\Gamma t / \hbar) \sin ^{2}\left(\frac{1}{2} \Omega_{E} t\right)$, is calculated without enhancement (curve $a$ ) and with enhancement (curve $b$ ). Curve $b$ with $\Gamma \rightarrow 4 \Gamma$ approximately reproduces the envelope of the measurement. The 19-ns shift of the peak response between $a$ and $b$ is due to the coherent enhancement. 
the earlier experiment of Ref. 2 with two reflecting YIG crystals, the speedup of the coherent decay shifts the peak response from 133 to 110 ns, again corresponding to a $4 \Gamma$ enhancement.

In summary, we have observed the delayed time spectrum of nuclear Bragg-scattered synchrotron radiation using a single-reflection geometry. The single-reflection geometry will make investigations possible in many other systems. We have also demonstrated how the quantumbeat pattern can be altered by a change in the coupling of the radiation to the hyperfine oscillators. By our simply rotating the applied external field, a new quantumbeat pattern was produced with more hyperfine information. Finally, our measurements illustrate the speedup of the coherent response, and the quantum-beat modulation of the coherent decay rate.

We acknowledge the continuous and helpful collaboration of Professor W. Tolksdorf and Dr. C. P. Klages of the Philips Forschungslaboratorium in Hamburg who produced the enriched YIG crystals. This work has been funded by the Bundesministerium für Forschung und Technologie (BMFT) under Contract No. 05270 GUI9. Partial support was also provided to one of us (J.P.H.) by the Welch Foundation through Grant No. C-1049, and by the National Science Foundation through Grant No. DMR-8608248.
${ }^{1}$ E. Gerdau, R. Rüffer, H. Winkler, W. Tolksdorf, C. P. Klages, and J. P. Hannon, Phys. Rev. Lett. 54, 835 (1985).

${ }^{2}$ E. Gerdau, R. Rüffer, R. Hollatz, and J. P. Hannon, Phys. Rev. Lett. 57, 1141 (1986).

${ }^{3}$ G. T. Trammell and J. P. Hannon, Phys. Rev. B 18, 165 (1978), and 19, 3835 (1979).

${ }^{4}$ H. Winkler, R. Eisberg, E. Alp, R. Rüffer, E. Gerdau, S. Lauer, A. X. Trautwein, M. Grodzicki, and A. Vera, Z Phys. B 49, 331 (1983).

${ }^{5} \mathrm{We}$ have dropped contributions from nondiagonal terms in the interaction matrix, which are responsible for the $\epsilon$ shifts

${ }^{6}$ All estimates given use the room-temperature data of YIG (Ref. 4 and Ref. 2).

${ }^{7}$ A more detailed discussion will be published elsewhere

${ }^{8}$ G. T. Trammell, in Chemical Effects on Nuclear Transformations (International Atomic Energy Agency, Vienna, 1961), Vol. 1, p. 75.

${ }^{9}$ J. P. Hannon and G. T. Trammell, Phys. Rev. 169, 315 (1968), and 186, 306 (1969).

${ }^{10}$ G. V. Smirnov, Yu. V. Shvyd'ko, and E. Realo, Pis'ma Zh. Eksp. Teor. Fiz. 39, 33 (1984) [JETP Lett. 39, 41 (1984)]; G. V. Smirnov and Yu. V. Shvyd'ko, Pis'ma Zh. Eksp. Teor. Fiz. 44, 431 (1986) [JETP Lett. 44, 556 (1986)].

${ }^{11}$ Yu. M. Kagan, A. F. Afanas'ev, and V. G. Kohn, Phys. Lett. 68A, 339 (1978); and J. Phys. C 12, 615 (1979).

12 J. P. Hannon, G. T. Trammell, M. Mueller, E. Gerdau, R. Rüffer, and H. Winkler, Phys. Rev. B 32, 6363 and 6374 (1985). 